\title{
HOLOMORPHIC EISENSTEIN SERIES WITH JACOBIAN TWISTS
}

\author{
LEV A. BORISOV
}

\begin{abstract}
For every point on the Jacobian of the modular curve $X_{0}(l)$ we define and study certain twisted holomorphic Eisenstein series. These are particular cases of a more general notion of twisted modular forms which correspond to sections on the modular curve $X_{1}(l)$ of the degree zero twists of line bundles of usual modular forms. We conjecture that a point on the Jacobian is rational if and only if the ratios of these twisted Eisenstein series of the same weights have rational coefficients.
\end{abstract}

\section{InTRODUCTION}

Let $X_{0}(l)$ denote the modular curve of level $l$. It is defined as the compactification of the quotient of the upper half plane $\mathcal{H}$ by the group $\Gamma_{0}(l)$ which consists of matrices $\left(\begin{array}{ll}a & b \\ c & d\end{array}\right) \in S L_{2}(\mathbb{Z})$ with $c \in l \mathbb{Z}$. We are interested in the Jacobian of $X_{0}(l)$. We associate to any point of this Jacobian a collection of holomorphic functions $E_{i, k ; h}(\tau)$ on the upper half plane $\mathcal{H}$, which we call $h$-twisted Eisenstein series. Here $k \geq 3$ is an integer, $i \in \mathbb{Z} / l \mathbb{Z}$, and $h=h(\tau)$ is a modular form of weight two for $\Gamma_{0}(l)$ which is related to the point of the Jacobian by period integrals.

More specifically, to any weight two form $h$ we associate a unitary character of $\Gamma_{0}(l)$ by

$$
\left(\begin{array}{ll}
a & b \\
c & d
\end{array}\right) \mapsto \exp \left(2 \pi \mathrm{i} \Re \int_{\mathrm{i} \infty}^{-\frac{d}{c}} h(s) d s\right) .
$$

Then we use this unitary character to modify a definition of some Eisenstein series. The unitarity of the character assures the convergence. It then turns out that the character and the series depend only on the point of the Jacobian, so one gets well-defined invariants of degree zero invertible sheaves on $X_{0}(l)$.

The author was partially supported by NSF grant DMS-0140172. 
We remark that twisted Eisenstein series and modular forms of this paper should not be confused with twisted Eisenstein series of Goldfeld and Gunnells, see Go and GG, which involve only an additive version of the character (1.1). However, a nonholomorphic analog of the Eisenstein series considered in this paper has already appeared in P.

The paper is organized as follows. In Section 2 we define the Eisenstein series twisted by modular forms $E_{k, i ; h}(\tau)$ and prove their convergence. We use the Dirichlet summation trick to get a formula for the Fourier coefficients of $E_{k, i ; h}$. In Section 3 we define a more general class of functions, called $h$-twisted modular forms. The definition is parallel to that of the usual modular forms, except for the extra character (1.1). We give a geometric interpretation of these twisted modular forms as sections of the twists of the line bundles of the usual modular forms on the modular curves by degree zero line bundles, which justifies our terminology. Section 4 extends the Petersson inner product to the twisted case. Section 5 contains the rationality Conjecture 5.1. as well as some highly circumstantial evidence for it. Finally, in Section 6 we list the open problems which currently by far outnumber the results.

Acknowledgements. I thank Paul Gunnells and Gautam Chinta who read the first version of the paper and suggested several useful references.

This paper is dedicated to the 60th birthday of Igor Dolgachev who was my PhD advisor at the University of Michigan in 1993-1996. His love of mathematics inspires everyone around him. It is also a safe bet that he can pinpoint exactly which 19th century mathematician has already proved all the results of this paper.

\section{EISENSTEIN SERIES TWISTED BY MODULAR FORMS}

In this section we introduce the main objects of interest. Let $l>0$ be a positive integer, which will be fixed throughout the paper. We will denote by $\tau$ the coordinate on the upper half plane $\mathcal{H}$ and will use the notation $q=\mathrm{e}^{2 \pi \mathrm{i} \tau}$. Let $h(\tau)=\sum_{n=1}^{\infty} a_{n} q^{n}$ be a cusp form of weight two for the congruence subgroup $\Gamma_{0}(l)$. Let $H(\tau)=\int_{\mathrm{i} \infty}^{\tau} h(s) d s$ be the antiderivative of $h$.

We first define the auxiliary series $\hat{E}_{k, \chi ; h}(\tau)$.

Definition 2.1. For a positive integer $k$, a character $\chi:(\mathbb{Z} / l \mathbb{Z})^{*} \rightarrow \mathbb{C}$ and $h$ as above, we define $h$-twisted Eisenstein series by

$$
\hat{E}_{k, \chi ; h}(\tau)=\sum_{\Gamma_{\infty} \backslash \Gamma_{0}(l)}(c \tau+d)^{-k} \chi(d) \mathrm{e}^{2 \pi \mathrm{i} \Re H\left(-\frac{d}{c}\right)} .
$$


Here $\Gamma_{\infty}$ is the infinite cyclic subgroup generated by $\tau \rightarrow \tau+1$.

Remark 2.2. In Definition 2.1 and throughout the rest of the paper we use the convention $H\left(-\frac{d}{0}\right)=0$. It is motivated by the fact that $-\frac{d}{c}$ is the preimage of $\tau=\mathrm{i} \infty$ under $\tau \rightarrow \frac{a \tau+b}{c \tau+d}$. So if $c=0$, this preimage is $\mathrm{i} \infty$, and $H(\mathrm{i} \infty)=0$ by the definition.

We can reformulate the above series as follows. The cosets of $\Gamma_{0}(l)$ over $\Gamma_{\infty}$ are given by pairs of integers $(c, d)$ with $c \equiv 0 \bmod l$ and $\operatorname{gcd}(c, d)=1$. Hence, we have

$$
\hat{E}_{k, \chi ; h}(\tau)=\sum_{c \in l \mathbb{Z}} \sum_{d \in \mathbb{Z}, \operatorname{gcd}(c, d)=1}(c \tau+d)^{-k} \chi(d) \mathrm{e}^{2 \pi \mathrm{i} \Re H\left(-\frac{d}{c}\right)} .
$$

We will be actually interested in a slight variation of this series, which is defined below.

Definition 2.3. We define

$$
E_{k, i ; h}(\tau)=\sum_{c \in l \mathbb{Z}} \sum_{d \in \mathbb{Z}, \operatorname{gcd}(l, d)=1}(c \tau+d)^{-k} \mathrm{e}^{-2 \pi \mathrm{i} \frac{d i}{l}} \mathrm{e}^{2 \pi \mathrm{i} \Re H\left(-\frac{d}{c}\right)} .
$$

Proposition 2.4. For $k \geq 3$, the series $E_{k, i ; h}(\tau)$ and $\hat{E}_{k, \chi ; h}(\tau)$ absolutely converge to holomorphic functions on the upper half plane.

Proof. The series of absolute values is $\sum_{(c, d) \in \mathbb{Z}^{2}-(0,0)}|c \tau+d|^{-k}$. It has $\leq$ (const) $R$ terms with $|c \tau+d| \in[R, R+1)$. Absolute convergence on compacts is also clear, which implies that the resulting sum is holomorphic.

Remark 2.5. Proposition 2.4 just barely fails for $k=2$ and fails quite miserably for $k=1$, which is perhaps the case of most interest.

By splitting the series $E_{k, i ; h}$ according to $\operatorname{gcd}(c, d)$ we observe that it is a linear combination of series of type $\hat{E}$ with coefficients that involve the values of Dedekind L-functions at $k$ as well as Gauss sums. To write it out explicitly, let us denote by $\chi_{1}, \ldots, \chi_{\phi(l)}$ the characters $(\mathbb{Z} / l \mathbb{Z})^{*} \rightarrow \mathbb{C}$

Proposition 2.6. For $k \geq 3$ there holds

$$
E_{k, i ; h}(\tau)=\frac{1}{\phi(l)} \sum_{j=1}^{\phi(l)}\left(\sum_{t \in(\mathbb{Z} / l \mathbb{Z})^{*}} \mathrm{e}^{-2 \pi \mathrm{i} \frac{t i}{l}} \chi_{j}^{-1}(t)\right) L\left(\chi_{j}, k\right) \hat{E}_{k, \chi_{j} ; h}(\tau)
$$

where $L(\chi, k)$ denotes the value of the Dedekind $L$-function at $k$, and $\phi$ is the Euler function. 
Proof. For any $i \in \mathbb{Z} / l \mathbb{Z}$ and $d$ coprime to $l$, we have

$$
\mathrm{e}^{-2 \pi \mathrm{i} \frac{d i}{l}}=\sum_{j=1}^{\phi(l)} \chi_{j}(d) r_{i, j}
$$

where

$$
r_{i, j}=\frac{1}{\phi(l)} \sum_{t \in(\mathbb{Z} / l \mathbb{Z})^{*}} \mathrm{e}^{-2 \pi \mathrm{i} \frac{t i}{l}} \chi_{j}^{-1}(t) .
$$

This implies

$$
\begin{aligned}
& E_{k, i ; h}(\tau)=\sum_{c \in l \mathbb{Z}} \sum_{d \in \mathbb{Z}, \operatorname{gcd}(l, d)=1} \sum_{j=1}^{\phi(l)}(c \tau+d)^{-k} r_{i, j} \chi_{j}(d) \mathrm{e}^{2 \pi \mathrm{i} \Re H\left(-\frac{d}{c}\right)} \\
& =\sum_{n \in \mathbb{Z}_{>0}, g c d(n, l)=1} \sum_{c_{1} \in l \mathbb{Z}} \sum_{d_{1} \in \mathbb{Z}, \operatorname{gcd}\left(c_{1}, d_{1}\right)=1} \sum_{j=1}^{\phi(l)} \\
& \left(c_{1} \tau+d_{1}\right)^{-k} n^{-k} r_{i, j} \chi_{j}\left(d_{1}\right) \chi_{j}(n) \mathrm{e}^{2 \pi \mathrm{i} \Re H\left(-\frac{d_{1}}{c_{1}}\right)} \\
& =\sum_{c_{1} \in l \mathbb{Z}} \sum_{d_{1} \in \mathbb{Z}, \operatorname{gcd}\left(c_{1}, d_{1}\right)=1} \sum_{j=1}^{\phi(l)}\left(c_{1} \tau+d_{1}\right)^{-k} r_{i, j} \chi_{j}\left(d_{1}\right) L\left(\chi_{j}, k\right) \mathrm{e}^{2 \pi \mathrm{i} \Re H\left(-\frac{d_{1}}{c_{1}}\right)}
\end{aligned}
$$

since $L\left(\chi_{j}, k\right)=\sum_{n>0, g c d(n, l)=1} n^{-k} \chi_{j}(n)$.

So we have

$$
\begin{gathered}
E_{k, i ; h}(\tau)=\sum_{j=1}^{\phi(l)} L\left(\chi_{j}, k\right) r_{i, j} \hat{E}_{k, \chi_{j} ; h}(\tau) \\
=\frac{1}{\phi(l)} \sum_{j=1}^{\phi(l)} \sum_{t \in(\mathbb{Z} / l \mathbb{Z})^{*}} L\left(\chi_{j}, k\right) \mathrm{e}^{-2 \pi \mathrm{i} \frac{t i}{l}} \chi_{j}^{-1}(t) \hat{E}_{k, \chi_{j} ; h}(\tau) .
\end{gathered}
$$

The absolute convergence that allows us to change the order of summation follows from the proof of Proposition 2.4

We will now use the Dirichlet summation formula to find Fourier expansions of $E_{k, i ; h}(\tau)$. Namely, we have for $k \geq 2$ and $\Im x>0$

$$
\sum_{n \in \mathbb{Z}}(x+n)^{-k}=\sum_{m>0} \frac{(-2 \pi \mathrm{i})^{k} m^{k-1}}{(k-1) !} \mathrm{e}^{2 \pi \mathrm{i} m x} .
$$

Equation (2.1) allows us to rewrite the formula for $E_{k, i ; h}(\tau)$. 
Proposition 2.7. For $k \geq 3$ we have

$$
\begin{gathered}
E_{k, i ; h}(\tau)=\sum_{d \in \mathbb{Z}, g c d(d, l)=1} d^{-k} \mathrm{e}^{-2 \pi \mathrm{i} \frac{d i}{l}}+\frac{(-2 \pi \mathrm{i})^{k}}{(k-1) !} \sum_{c \in l \mathbb{Z}>0} \sum_{d_{0} \in(\mathbb{Z} / c \mathbb{Z}), g c d\left(d_{0}, l\right)=1} \\
c^{-k} \sum_{m>0} m^{k-1} q^{m}\left(\mathrm{e}^{-2 \pi \mathrm{i} \frac{d_{0} i}{l}}+(-1)^{k} \mathrm{e}^{2 \pi \mathrm{i} \frac{d_{0} i}{l}}\right) \mathrm{e}^{2 \pi \mathrm{i}\left(m \frac{d_{0}}{c}+\Re H\left(-\frac{d_{0}}{c}\right)\right)}
\end{gathered}
$$

where $q=\mathrm{e}^{2 \pi \mathrm{i} \tau}$.

Proof. The terms with $c=0$ correspond to the $q^{0}$ term. Here we use the convention $H\left(-\frac{d}{0}\right)=0$ of Remark 2.2.

For a given value of $c>0$, the possible values of $d$ are given by $d=d_{0}+n c$ with $0<d_{0}<c, \operatorname{gcd}\left(d_{0}, l\right)=1$ and $n \in \mathbb{Z}$. For each $d_{0}$ the value of $\Re H\left(-\frac{d}{c}\right)$ is independent of $n$. Indeed, one easily sees that $H(\tau+1)=H(\tau)$, since $h(\tau)$ was a cusp form. Then one uses (2.1) to sum $\left(c \tau+d_{0}+n c\right)^{-k}=c^{-k}\left(\tau+\frac{d_{0}}{c}+n\right)^{-k}$ over all integer $n$.

Finally, we notice that terms for $c<0$ can be obtained for those for $|c|>0$ by changing $d_{0}$ to $-d_{0}$. Consequently, we sum for $c>0$ only by taking into account terms with $(-c)$.

As a corollary, we observe that $E_{k, i ; h}(\tau)$ has a Fourier expansion.

Corollary 2.8. For $k \geq 3$ one has

$$
E_{k, i ; h}(\tau)=R_{0}+\sum_{m>0} R_{m} q^{m}
$$

where

$$
\begin{aligned}
R_{m}= & \frac{(-2 \pi \mathrm{i})^{k} m^{k-1}}{(k-1) !} \sum_{\substack{c \in l \mathbb{Z}>0 \\
d_{0} \in(\mathbb{Z} / c \mathbb{Z}), g c d\left(d_{0}, l\right)=1}} \sum_{c^{-k}\left(\mathrm{e}^{-2 \pi \mathrm{i} \frac{d_{0} i}{l}}+(-1)^{k} \mathrm{e}^{2 \pi \mathrm{i} \frac{d_{0} i}{l}}\right) \mathrm{e}^{2 \pi \mathrm{i}\left(m \frac{d_{0}}{c}+\Re H\left(-\frac{d_{0}}{c}\right)\right)}, m>0} \sum_{d \in \mathbb{Z}, g c d(d, l)=1} d^{-k} \mathrm{e}^{-2 \pi \mathrm{i} \frac{d i}{l}} .
\end{aligned}
$$

Proof. Follows from Proposition 2.7

Remark 2.9. It is instructive to see what happens for $h=H=0$. Then for a fixed $c=c_{0} l$ the sum over $d_{0}$ that are the same $\bmod l$ is zero unless $m$ is divisible by $c_{0}$. Indeed, for a given $d_{0} \bmod l$ we are summing over $d_{0}=s+r l \bmod c_{0} l$ with $0 \leq r<c_{0}$. The resulting sum of $\mathrm{e}^{2 \pi \mathrm{i} m \frac{r}{c_{0}}}$ is zero unless $c_{0} \mid m$. This gives

$$
R_{m}=\frac{(-2 \pi \mathrm{i})^{k} m^{k-1}}{l^{k}(k-1) !} \sum_{c_{0} \mid m} c_{0}^{-k} \sum_{\substack{d_{0} \in\left(\mathbb{Z} / c_{0} l \mathbb{Z}\right), g c d\left(d_{0}, l\right)=1}}\left(\mathrm{e}^{-2 \pi \mathrm{i} d_{0}\left(\frac{i}{l}-\frac{m}{l c_{0}}\right)}+(-1)^{k} \mathrm{e}^{2 \pi \mathrm{i} d_{0}\left(\frac{i}{l}+\frac{m}{l c_{0}}\right)}\right)
$$




$$
\begin{gathered}
=\frac{(-2 \pi \mathrm{i})^{k} m^{k-1}}{l^{k}(k-1) !} \sum_{c_{0} \mid m} c_{0}^{-k} c_{0} \sum_{j \in(\mathbb{Z} / l \mathbb{Z})^{*}}\left(\mathrm{e}^{-2 \pi \mathrm{i} j\left(\frac{i-\frac{m}{c_{0}}}{l}\right)}+(-1)^{k} \mathrm{e}^{2 \pi \mathrm{i} j\left(\frac{i+\frac{m}{c_{0}}}{l}\right)}\right) \\
=\frac{(-2 \pi \mathrm{i})^{k}}{l^{k}(k-1) !} \sum_{r \mid m} r^{k-1} \sum_{j \in(\mathbb{Z} / l \mathbb{Z})^{*}}\left(\mathrm{e}^{-2 \pi \mathrm{i} \frac{j(i-r)}{l}}+(-1)^{k} \mathrm{e}^{\left.2 \pi \mathrm{i} \frac{j(i+r)}{l}\right)}\right) .
\end{gathered}
$$

This can be recognized as the coefficient by $q^{m}$ of a linear combination of the Eisenstein series from [BG2]. Indeed, it is the sum over the divisors $r$ of $m$ of an odd $(\bmod l)$-polynomial function of $r$ of degree $(k-1)$, see [BG2]. In the particular case of prime $l$ we get

$$
\frac{(-2 \pi \mathrm{i})^{k}}{l^{k-1}(k-1) !} \sum_{r \mid m} r^{k-1} \sum_{j \in(\mathbb{Z} / l \mathbb{Z})^{*}}\left(\delta_{r}^{i \bmod l}+(-1)^{k} \delta_{r}^{-i \bmod l}-\frac{2}{l} \delta_{k}^{0 \bmod 2}\right)
$$

where $\delta$ is the Kronecker symbol.

Remark 2.10. It is easy to see that the coefficients by $q^{m}$ of $\frac{1}{(2 \pi \mathrm{i})^{k}} E_{k, i ; 0}$ are rational for $m>0$. Indeed, $\sum_{j \in(\mathbb{Z} / l \mathbb{Z})^{*}}\left(\mathrm{e}^{-2 \pi \mathrm{i} \frac{j(i-r)}{l}}+(-1)^{k} \mathrm{e}^{\left.2 \pi \mathrm{i} \frac{j(i+r)}{l}\right)}\right)$ is invariant under the Galois group of $\mathbb{Q}\left[\mathrm{e}^{2 \pi \mathrm{i} / l}\right] \supset \mathbb{Q}$. Since the space of Eisenstein series has a basis whose elements have all Fourier coefficients in $\mathbb{Q}$, the $q^{0}$ coefficients of $\frac{1}{(2 \pi \mathrm{i})^{k}} E_{k, i ; 0}$ are rational as well. This is the main reason we prefer $\frac{1}{(2 \pi \mathrm{i})^{k}} E_{k, i ; h}$ to their close relatives $\frac{1}{(2 \pi \mathrm{i})^{k}} \hat{E}_{k, \chi ; h}$ that do not have rational Fourier coefficients even in the untwisted case.

Armed with the formulas for its Fourier coefficients, we can now try to define $E_{k, i ; h}$ formally by their Fourier expansions in the interesting cases $k=1$ and $k=2$. This approach works in the untwisted case, but fails in general. Indeed, let us analyze the convergence of the series in Corollary 2.8. We rewrite it as

$$
\sum_{c_{0} \in \mathbb{Z}_{>0}} \sum_{d_{0} \in\left(\mathbb{Z} / c_{0} l \mathbb{Z}\right), g c d\left(d_{0}, l\right)=1} c_{0}^{-k}\left(\mathrm{e}^{-2 \pi \mathrm{i} \frac{d_{0} i}{l}}+(-1)^{k} \mathrm{e}^{2 \pi \mathrm{i} \frac{d_{0} i}{l}}\right) \mathrm{e}^{2 \pi \mathrm{i}\left(m \frac{d_{0}}{c}+\Re H\left(-\frac{d_{0}}{c_{0} l}\right)\right)} .
$$

For a given $d_{0} \bmod l=j \bmod l$, we are dealing with convergence of

$$
\sum_{c_{0} \in \mathbb{Z}_{>0}} \sum_{d_{0} \in\left(\mathbb{Z} / c_{0} l \mathbb{Z}\right), d_{0} \equiv j \bmod l} c_{0}^{-k} \mathrm{e}^{2 \pi \mathrm{i}\left(m \frac{d_{0}}{c}+\Re H\left(-\frac{d_{0}}{c_{0} l}\right)\right)} .
$$

There is no absolute convergence of the double sum, even in the $k=2$ case. However, as the nontwisted case $H=0$ suggests, one can try to look at the finite sums for fixed $c_{0}$ first and then look at the resulting series. It is conceivable that this series will converge absolutely, at least for $k=2$. Indeed, for small $h$ the values of the sums may be close to those for $h=0$ where all but a finite number are zero. But this is definitely not a proof. For instance, the values of $\Re H\left(-\frac{d_{0}}{c_{0} l}\right)$ are not 
bounded, although one can show that they grow at most logarithmically in $c_{0}$.

\section{TWISTED MODULAR FORMS}

In this section we put the definition of the $h$-twisted Eisenstein series into a more general context of the $h$-twisted modular forms. We denote by $X_{0}(l)$ and $X_{1}(l)$ the modular curves for the congruence subgroups $\Gamma_{0}(l)$ and $\Gamma_{1}(l)$. The group $\Gamma_{1}(l)$ is the subgroup of $\Gamma_{0}(l)$ whose diagonal elements are $1 \bmod l$.

Definition 3.1. An $h$-twisted modular form of weight $k$ with respect to $\Gamma_{1}(l)$ is a holomorphic function $f$ on the upper half plane, which satisfies

(1) For any $\left(\begin{array}{ll}a & b \\ c & d\end{array}\right) \in \Gamma_{1}(l)$ there holds

$$
f\left(\frac{a \tau+b}{c \tau+d}\right)=(c \tau+d)^{k} f(\tau) \mathrm{e}^{-2 \pi \mathrm{i} \Re H\left(-\frac{d}{c}\right)} .
$$

(2) For any $\left(\begin{array}{ll}a & b \\ c & d\end{array}\right) \in S L_{2}(\mathbb{Z})$ the function

$$
f\left(\frac{a \tau+b}{c \tau+d}\right)(c \tau+d)^{-k}
$$

is bounded near i $\infty$.

We immediately see that $E_{k, i ; h}(\tau)$ and $\hat{E}_{k, \chi ; h}(\tau)$ are $h$-twisted modular forms of level $l$.

Proposition 3.2. For $k \geq 3$, the functions $E_{k, i ; h}(\tau)$ and $\hat{E}_{k, \chi ; h}(\tau)$ are $h$-twisted modular form of weight $k$ for $\Gamma_{1}(l)$.

Proof. We will first check the transformation properties of $E_{k, i ; h}(\tau)$ under $\tau \rightarrow \frac{a \tau+b}{c \tau+d}$. If the sum in the definition of $E_{k, i ; h}$ is taken over $\left(c_{1}, d_{1}\right)$, then we can rewrite the sum for $E_{k, i ; h}\left(\frac{a \tau+b}{c \tau+d}\right)$ in terms of the sum over $\left(c_{2}, d_{2}\right)=\left(c_{1} a+d_{1} c, c_{1} b+d_{1} d\right)$ as

$$
E_{k, i ; h}\left(\frac{a \tau+b}{c \tau+d}\right)=\sum_{c_{2} \in l \mathbb{Z}} \sum_{\substack{d_{2} \in \mathbb{Z}, g c d\left(d_{2}, l\right)=1}}\left(c_{2} \tau+d_{2}\right)^{-k}(c \tau+d)^{k} \mathrm{e}^{-2 \pi \mathrm{i} \frac{d_{1} i}{l}} \mathrm{e}^{2 \pi \mathrm{i} \Re H\left(-\frac{d_{1}}{c_{1}}\right)} .
$$

We notice that $d_{1} \equiv d_{2} \bmod l$, so the first exponential term equals $\mathrm{e}^{-2 \pi \mathrm{i} \frac{d_{2} i}{l}}$. It then suffices to show that $H\left(-\frac{d_{1}}{c_{1}}\right)=H\left(-\frac{d_{2}}{c_{2}}\right)-H\left(-\frac{d}{c}\right)$. 
Since $H(\tau)$ is the antiderivative of a weight two $\Gamma_{0}(l)$ modular form $h$, the transformation properties of $h(\tau)$ imply

$$
H\left(\frac{-d \tau+b}{c \tau-a}\right)=H(\tau)+C
$$

where $C$ is independent of $\tau$. By plugging in $\tau=\mathrm{i} \infty$, we calculate $C$ to get $H\left(\frac{d_{1} \tau+b_{1}}{c_{1} \tau-a_{1}}\right)=H(\tau)+H\left(-\frac{d}{c}\right)$. Then we plug in $\tau=-\frac{d_{1}}{c_{1}}$ to get $H\left(-\frac{d_{2}}{c_{2}}\right)=H\left(-\frac{d_{1}}{c_{1}}\right)+H\left(-\frac{d}{c}\right)$.

We now check that for any $\left(\begin{array}{ll}a & b \\ c & d\end{array}\right) \in S L_{2}(\mathbb{Z})$ the function

$$
E_{i, k ; h}\left(\frac{a \tau+b}{c \tau+d}\right)(c \tau+d)^{-k}
$$

is bounded near $i \infty$. We have

$$
E_{k, i ; h}\left(\frac{a \tau+b}{c \tau+d}\right)(c \tau+d)^{-k}=\sum_{c_{1} \in l \mathbb{Z}} \sum_{\substack{d_{1} \in \mathbb{Z}, g c d\left(d_{1}, l\right)=1}}\left(c_{2} \tau+d_{2}\right)^{-k} \mathrm{e}^{-2 \pi \mathrm{i} \frac{d_{1} i}{l}} \mathrm{e}^{2 \pi \mathrm{i} \Re H\left(-\frac{d_{1}}{c_{1}}\right)} .
$$

Each term is bounded by $\left|c_{2} \tau+d_{2}\right|^{-k}$. We extend the summation set to sum over $\left(c_{2}, d_{2}\right) \in \mathbb{Z}^{2}-\{(0,0)\}$ and assume $\Im \tau \geq 1$. Then $\left|c_{2} \tau+d_{2}\right| \geq 1$, and we consider the subsums for $\left|c_{2} \tau+d_{2}\right| \in[m, m+1)$ for all positive integer $m$. If we slice the annulus $|z| \in[m, m+1)$ into $m$ equal pieces according to the polar angle, we see that each slice contains at most a constant number of elements of $\mathbb{Z} \tau+\mathbb{Z}$, since this lattice has no elements of length less than one. Importantly, this constant $C$ is independent of $\tau$. This gives

$$
\sum_{\left(c_{2}, d_{2}\right)}\left|c_{2} \tau+d_{2}\right|^{-k} \leq C \sum_{m>0} m^{-k+1}
$$

which converges for $k \geq 3$.

The argument for $\hat{E}_{k, \chi ; h}$ is similar and is left to the reader.

We can extend the Proposition 3.2 to describe the transformation properties of $E_{k, i ; h}$ and $\hat{E}_{k, \chi ; h}$ under $\Gamma_{0}(l)$.

Proposition 3.3. For $k \geq 3$, for every $\tau \rightarrow \frac{a \tau+b}{c \tau+d} \in \Gamma_{0}(l)$ one has

$$
\begin{gathered}
E_{k, i ; h}\left(\frac{a \tau+b}{c \tau+d}\right)=E_{k, a i ; h}(\tau)(c \tau+d)^{k} \mathrm{e}^{-2 \pi \mathrm{i} \Re H\left(-\frac{d}{c}\right)} \\
E_{k, \chi ; h}\left(\frac{a \tau+b}{c \tau+d}\right)=E_{k, \chi ; h}(\tau)(c \tau+d)^{k} \chi(a) \mathrm{e}^{-2 \pi \mathrm{i} \Re H\left(-\frac{d}{c}\right)} .
\end{gathered}
$$


Proof. The argument for $E_{k, i ; h}$ follows that of Proposition 3.2. The only difference is what happens to the term $\mathrm{e}^{-2 \pi \mathrm{i} \frac{d_{1} i}{l}}$, where one now observes that $d_{2} \equiv d_{1} d \bmod l$, so $d_{1} \equiv a d_{2} \bmod l$.

The argument for $\hat{E}_{k, \chi ; h}$ is similar and is left to the reader.

Remark 3.4. The space of $h$-twisted modular forms of weight $k$ and with respect to $\Gamma_{1}(l)$ admits a natural action of $\Gamma_{0}(l) / \Gamma_{1}(l) \cong(\mathbb{Z} / l \mathbb{Z})^{*}$. It sends $f(\tau)$ to

$$
f\left(\frac{a \tau+b}{c \tau+d}\right)(c \tau+d)^{-k} \mathrm{e}^{2 \pi \mathrm{i} \Re H\left(-\frac{d}{c}\right)} .
$$

The eigenvectors of this action will be called $h$-twisted modular forms of nebentypus $\chi$. They satisfy

$$
f\left(\frac{a \tau+b}{c \tau+d}\right)=f(\tau)(c \tau+d)^{k} \chi(a) \mathrm{e}^{-2 \pi \mathrm{i} \Re H\left(-\frac{d}{c}\right)}
$$

for $\left(\begin{array}{ll}a & b \\ c & d\end{array}\right)$ in $\Gamma_{0}(l)$. We notice that $\hat{E}_{k, \chi ; h}$ have nebentypus $\chi$.

Remark 3.5. We will call the nebentypus $\chi=1$ the $h$-twisted $\Gamma_{0}(l)$ modular forms. For example, $E_{k, 0 ; h}(\tau)$ is one such form for $k \geq 3$. As in the usual case, the action of $\mathbf{- 1} \in \Gamma_{0}(l)$ causes the spaces of odd weight to be zero.

It is clear that for a given $h$, the space of $h$-twisted modular forms for $\Gamma_{1}(l)$ is a graded module over the ring of modular forms for $\Gamma_{1}(l)$. In what follows we give a geometric interpretation of this space. We can safely assume $l \geq 3$ so the action of $\Gamma_{1}(l)$ on the upper half plane is free. We denote by $X_{1}(l)$ the corresponding modular curve which is obtained by the compactification of its open subset $\mathcal{H} / \Gamma_{1}(l)$. There is a line bundle $\mathcal{L}$ of weight one modular forms on $X_{1}(l)$, such that the space $M_{k}\left(\Gamma_{1}(l)\right)$ of usual modular forms of weight $k$ is naturally identified with the space of global sections $H^{0}\left(X_{1}(l), \mathcal{L}^{\otimes k}\right)$.

Proposition 3.6. For each $h$ there is a degree zero invertible $\mathcal{F}=\mathcal{F}(h)$ such that the space of weight $k$ h-twisted modular forms of level $n$ is naturally identified with $H^{0}\left(X_{1}(l), \mathcal{F} \otimes \mathcal{L}^{\otimes k}\right)$.

Proof. For an $h$-twisted modular form $f$ we define the order of zero of $f$ at a cusp to be the exponent in the corresponding Fourier expansion. Combined with the zeroes in the open set $\mathcal{H} / \Gamma_{1}(l)$, they give a divisor on $X_{1}(l)$ which we denote by $\operatorname{Div}(f)$.

We observe that the divisor class of Div $(f)$ depends only on $h$ and $k$. Indeed, the ratio $f(\tau)=\frac{f_{1}(\tau)}{f_{2}(\tau)}$ of two weight $k h$-twisted modular forms is a $\Gamma_{1}(l)$ modular function. It remains to observe that $\operatorname{Div}(f)=$ 
$\operatorname{Div}\left(f_{1}\right)-\operatorname{Div}\left(f_{2}\right)$. More generally, for $h$-twisted forms $f_{i}$ of weights $k_{i}$ one has $\operatorname{Div}\left(f_{1}\right)-\operatorname{Div}\left(f_{2}\right) \sim\left(k_{1}-k_{2}\right) \operatorname{Div}(L)$ where $\operatorname{Div}(\mathcal{L})$ is the divisor of any (meromorphic) modular form of weight 1 . We define $\mathcal{F}$ as the invertible sheaf that corresponds to $\operatorname{Div}\left(E_{4,0 ; h}\right)-\operatorname{Div}\left(E_{4,0 ; 0}\right)$.

Every $h$-twisted modular form of weight $k$ gives a global section of $\mathcal{F} \otimes \mathcal{L}^{\otimes k}$. Conversely, given a global section of $\mathcal{F} \otimes \mathcal{L}^{\otimes k}$, one can write it as a product of $E_{4,0 ; h}(\tau)$ and a modular function on $X_{1}(l)$. This product is easily seen to be a holomorphic function on $\mathcal{H}$ that satisfies the definition of the $h$-twisted modular form of weight $k$.

It remains to see that the degree of $\mathcal{F}$ is zero. Consider a ratio of an $h$-twisted and a usual form of the same weight $k$, which we can assume to be nonzero at the cusps. It is a meromorphic function $g(\tau)$ on the upper half plane, which is bounded at the cusps. To find the number of its zeroes and poles in the fundamental domain, one needs to integrate to find the sum of residues of $d \log g(\tau)$ in it. Transformation properties of $g$ imply that $d \log g(\tau)$ is a meromorphic differential form on $X_{1}(l)$. Consequently, the sum of its residues is zero, which leads to $\operatorname{deg} \mathcal{F}=0$, as desired.

Proposition 3.7. In the notations above, the invertible sheaf $\mathcal{F}(h)$ is invariant under the action of $\Gamma_{0}(l) / \Gamma_{1}(l)$. Moreover, it admits a natural linearization for this action.

Proof. The key point here is that $h(\tau)$ was a $\Gamma_{0}(l)$ modular form. The linearization is provided by the action of $\Gamma_{0}(l) / \Gamma_{1}(l)$ from Remark 3.4.

Remark 3.8. We may as well talk about the invertible sheaf $\mathcal{F}_{0}=$ $\mathcal{F}_{0}(h)$ of degree zero on the modular curve $X_{0}(l)$. It can be either defined as the $\Gamma_{0}(l) / \Gamma_{1}(l)$-invariant part of the pushforward $\pi_{*} \mathcal{F}$ for the quotient map $\pi: X_{1}(l) \rightarrow X_{0}(l)$, or as a sheaf that corresponds to the divisor $\operatorname{Div}\left(E_{4,0 ; h}\right)-\operatorname{Div}\left(E_{4,0 ; 0}\right)$ on $X_{0}(l)$. We have $\pi^{*} \mathcal{F}_{0} \cong \mathcal{F}$.

Remark 3.9. The operation of tensoring by an invertible sheaf is commonly referred to as twisting, which justifies our terminology. Unfortunately, this term is already used in [GG] in the context of Eisenstein series. We hope that this does not lead to a confusion, since the series we construct in this paper are quite different from those in [GG].

Theorem 3.10. Any degree zero invertible sheaf $\mathcal{F}_{0}$ on $X_{0}(l)$ is isomorphic to $\mathcal{F}_{0}(h)$ of Remark 3.8 for some weight two $\Gamma_{0}(l)$ cusp form $h$.

Proof. The map $h \mapsto \mathcal{F}_{0}(h)$ can be described in terms of the periods. Let $r$ be the genus of $X_{0}(l)$, which we assume to be positive. Denote 
by $K_{X_{0}(l)}$ the sheaf of holomorphic 1 -forms on $X_{0}(l)$. The Jacobian of $X_{0}(l)$ is isomorphic to the quotient of $\mathbb{C}^{r}=H^{0}\left(X_{0}(l), K_{X_{0}(l)}\right)^{\vee}$ by the lattice of periods, defined as the image of $H_{1}\left(X_{0}(l), \mathbb{Z}\right)$ via integration, see $[\mathrm{GH}]$. We would like to calculate the point of the Jacobian that corresponds to $\mathcal{F}_{0}(h)$ in these terms. This means that for any $g \in$ $H^{0}\left(X_{0}(l), K_{X_{0}(l)}\right)$ we would like to find the integral of $g$ from zeroes of some $h$-twisted weight $k$ form $f_{h}$ to zeroes of some usual weight $k$ form $f$, up to the lattice of periods.

By an easy Riemann-Roch calculation, we can safely assume that $f_{h}$ and $f$ do not vanish at the extra symmetry points or cusps, where by extra symmetry points we mean those that are equivalent to i or $\mathrm{e}^{2 \pi \mathrm{i} / 3}$ modulo $S L_{2}(\mathbb{Z})$. Let $G(\tau)=\int_{\mathrm{i} \infty}^{\tau} g(s) d s$ be an antiderivative of $g$. In order to find the integral of $g$ from the zeroes of $f$ to the zeroes of $f_{h}$, we need to find the integral

$$
I=\frac{1}{2 \pi \mathrm{i}} \int_{\partial \mathcal{H} / \Gamma_{0}(l)} G(\tau)\left(\frac{f_{h}^{\prime}(\tau)}{f_{h}(\tau)}-\frac{f^{\prime}(\tau)}{f(\tau)}\right) d \tau
$$

over the boundary of a fundamental domain of $\Gamma_{0}(l)$ action on $\mathcal{H}$. Different choices of fundamental domains will give answers that differ by periods of $g$.

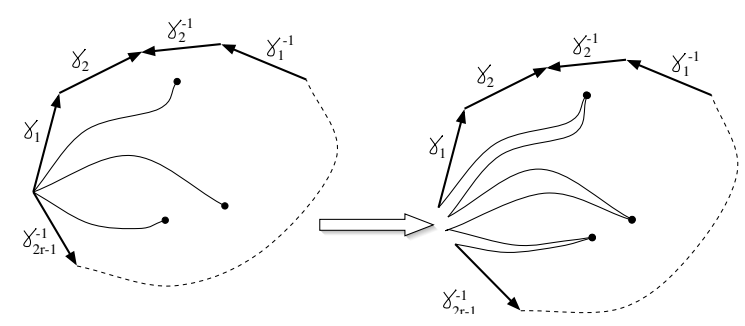

FiguRE 1.

Let us pick a fundamental domain as follows. Pick a point $p \in X_{0}(l)$ and cut $X_{0}(l)$ to represent it by a $4 r$-gon with pairs of sides identified in the usual manner, such that all vertices map to $p$. We will assume that the cusps, the points of extra symmetry, as well as the zeroes of $f_{h}$ and $f$ lie in the interior of this polygon. We will draw cuts from a vertex of the $4 r$-gon to the cusps and extra symmetry points, see Figure 1. We can represent the resulting $4 r$-gon without these extra cuts by a region in the upper half plane by picking a preimage of a point in the middle and extending all the paths from it by continuity. This will have an effect of opening up the cuts to the cusps and to those extra symmetry 
points whose isotropy subgroup in $\Gamma_{0}(l)$ is bigger than just $\{ \pm 1\}$, see Figure 1.

The boundary arcs of the resulting fundamental domain $\mathcal{D}$ are paired up according to the gluing required to make $X_{0}(l)$. These arcs will then be related by a coordinate transformation $\tau \rightarrow \frac{a \tau+b}{c \tau+d}$ from $\Gamma_{0}(l)$. We first investigate pairs of paths that go to the cusps or symmetry points of $X_{0}(l)$. Whether or not these cuts open up, the values of $G(\tau)\left(\frac{f_{h}^{\prime}(\tau)}{f_{h}(\tau)}-\right.$ $\left.\frac{f^{\prime}(\tau)}{f(\tau)}\right)$ at the opposite sides of the cut are the same. Indeed, $G\left(\frac{a \tau+b}{c \tau+d}\right)=$ $G(\tau)$ for any $\tau \rightarrow \frac{a \tau+b}{c \tau+d}$ from $\Gamma_{0}(l)$ that has a fixed point. Similar statement holds for $H(\tau)$, and the transformation properties of $f_{h}$ and $f$ show that $d \log f_{h}-d \log f$ is unchanged under such transformations.

So we are left to integrate over the $\operatorname{arcs} p_{j} \rightarrow q_{j}$ of the boundary that correspond to the sides of the original $4 r$-gon. Pair them up according to the gluings. The two $\operatorname{arcs} \gamma_{j}$ and $\gamma_{j}^{-1}$ (see Figure 1) differ by $\tau \rightarrow \frac{a_{j} \tau+b_{j}}{c_{j} \tau+d_{j}}$ from $\Gamma_{0}(l)$, and we are looking at

$$
\begin{gathered}
I=\frac{1}{2 \pi \mathrm{i}} \sum_{j=1}^{2 r} \int_{p_{j}}^{q_{j}}\left(G\left(\frac{a_{j} \tau+b_{j}}{c_{j} \tau+d_{j}}\right)\left(\frac{f_{h}^{\prime}\left(\frac{a_{j} \tau+b_{j}}{c_{j} \tau+d_{j}}\right)}{f_{h}\left(\frac{a_{j} \tau+b_{j}}{c_{j} \tau+d_{j}}\right)}-\frac{f^{\prime}\left(\frac{a_{j} \tau+b_{j}}{c_{j} \tau+d_{j}}\right)}{f\left(\frac{a_{j} \tau+b_{j}}{c_{j} \tau+d_{j}}\right)}\right)\left(c_{j} \tau+d_{j}\right)^{-2}\right. \\
\left.-G(\tau)\left(\frac{f_{h}^{\prime}(\tau)}{f_{h}(\tau)}-\frac{f^{\prime}(\tau)}{f(\tau)}\right)\right) d \tau
\end{gathered}
$$

Transformation properties of $G, f_{h}$ and $f$ allow us to simplify the integrand to be

$$
\begin{gathered}
\left(\left(G(\tau)+\alpha_{j}\right)\left(\frac{f_{h}^{\prime}(\tau)}{f_{h}(\tau)}-\frac{f^{\prime}(\tau)}{f(\tau)}\right)-G(\tau)\left(\frac{f_{h}^{\prime}(\tau)}{f_{h}(\tau)}-\frac{f^{\prime}(\tau)}{f(\tau)}\right)\right) \\
=\alpha_{j}\left(\frac{f_{h}^{\prime}(\tau)}{f_{h}(\tau)}-\frac{f^{\prime}(\tau)}{f(\tau)}\right)
\end{gathered}
$$

where $\alpha_{j}=G\left(\frac{a_{j} \tau+b_{j}}{c_{j} \tau+d_{j}}\right)-G(\tau)$. Hence we get

$$
\begin{gathered}
I=\frac{1}{2 \pi \mathrm{i}} \sum_{j=1}^{2 r} \int_{p_{j}}^{q_{j}} \alpha_{j}\left(\left(\frac{f_{h}^{\prime}(\tau)}{f_{h}(\tau)}-\frac{f^{\prime}(\tau)}{f(\tau)}\right) d \tau=\left.\frac{1}{2 \pi \mathrm{i}} \sum_{j=1}^{2 r} \alpha_{j} \log \left(\frac{f_{h}(\tau)}{f(\tau)}\right)\right|_{p_{j}} ^{q_{j}}\right. \\
=-\sum_{j=1}^{2 r} \alpha_{j} \Re H\left(-\frac{\hat{d}_{j}}{\hat{c}_{j}}\right) \bmod \text { periods }
\end{gathered}
$$

where $\tau \rightarrow \frac{\hat{a}_{j} \tau+\hat{b}_{j}}{\hat{c}_{j} \tau+\hat{d}_{j}}$ is an element of $\Gamma_{0}(l)$ that sends $p_{j} \mapsto q_{j}$. Indeed, the transformation properties of $f_{h}$ and $f$ lead to the transformation properties of the logarithms, up to integer constants that are independent of $g$, and $\alpha_{i}$ are the period integrals. 
We observe that $\alpha_{j}$ are the period integrals of $g(\tau) d \tau$ over the standard symplectic basis of $H_{1}\left(X_{0}(l), \mathbb{Z}\right)$, as are $H\left(-\frac{\hat{d}_{j}}{\hat{c}_{j}}\right)$ are the integrals of $h(\tau) d \tau$ over the same basis. Indeed, $H\left(\frac{\hat{a}_{j} \tau+\hat{b}_{j}}{\hat{c}_{j} \tau+\hat{d}_{j}}\right)=H(\tau)-H\left(-\frac{\hat{d}_{j}}{\hat{c}_{j}}\right)$ in view of $H(\mathrm{i} \infty)=0$, so $-H\left(-\frac{\hat{d}_{j}}{\hat{c}_{j}}\right)=H\left(q_{j}\right)-H\left(p_{j}\right)=\int_{p_{j}}^{q_{j}} h(\tau) d \tau$. To show that any point of the Jacobian can be given by $\mathcal{F}_{h}$, it is enough to show that the pairing (of real vector spaces)

$$
H_{1}\left(X_{0}(l), \mathbb{R}\right) \times H^{0}\left(X_{0}(l), K_{X_{0}(l)}\right) \rightarrow \mathbb{R},(\gamma, w) \mapsto \Re \int_{\gamma} w
$$

is nondegenerate. This is a standard general fact, which is true for any projective curve $X$, but we provide the argument below for the benefit of the reader.

By picking a basis $\left(w_{1}, \ldots, w_{r}\right)$ dual to a half of a symplectic basis $\left(\gamma_{1}, \ldots, \gamma_{2 r}\right)$, the period matrix $\Omega=\left(\int_{\gamma_{j}} w_{i}\right)$ is given by $\left(\mathbf{I}_{r}, Z\right)$, where $\Im Z$ is positive definite (see [GH]). If $\int_{\gamma_{j}} w$ is purely imaginary for all $j$, then we first see that $w=\sum_{i=1}^{r} \lambda_{i} w_{i}$ with purely imaginary $\lambda_{i}$. Then $\left(\lambda_{i}\right)$ lies in the kernel of $Z$, so $\Im Z>0$ implies $\left(\lambda_{i}\right)=\mathbf{0}$.

Corollary 3.11. Two weight two cusp forms $h_{1}$ and $h_{2}$ have $\mathcal{F}_{1} \cong$ $\mathcal{F}_{2}$ if and only if $\Re\left(H_{1}\left(-\frac{d}{c}\right)-H_{2}\left(-\frac{d}{c}\right)\right) \in \mathbb{Z}$ for all $c \in l \mathbb{Z}$ and $d \in$ $\mathbb{Z}, \operatorname{gcd}(d, l)=1$. In particular, the Eisenstein series $E_{k, i ; h}$ depend only on the point of the Jacobian.

Proof. The if part is clear, since in this case the Eisenstein series are identical.

To see the only if part, we observe that in the proof of Theorem 3.10, the sheaf $\mathcal{F}$ is given by a linear combination of the periods with coefficients $\Re H\left(-\frac{\hat{d}_{j}}{\hat{c}_{j}}\right), j=1, \ldots, 2 r$. If $\mathcal{F}_{1} \cong \mathcal{F}_{2}$, then

$$
\Re\left(H_{1}\left(-\frac{\hat{d}_{j}}{\hat{c}_{j}}\right)-H_{2}\left(-\frac{\hat{d}_{j}}{\hat{c}_{j}}\right)\right) \in \mathbb{Z}
$$

for $j=1, \ldots, 2 r$. For any $\left(\begin{array}{ll}a & b \\ c & d\end{array}\right)$ in $\Gamma_{0}(l)$, the values of $\Re H_{1}\left(-\frac{d}{c}\right)-$ $\Re H_{2}\left(-\frac{d}{c}\right)$ are integer linear combinations of the periods in (3.1). Indeed, this follows from the fact that (3.1) gives integrals of $\left(h_{1}(\tau)-\right.$ $\left.h_{2}(\tau)\right) d \tau$ over a basis of $H_{1}\left(X_{0}(l), \mathbb{Z}\right)$. Finally, any ratio $-\frac{d}{c}$ with $c \in l \mathbb{Z}$ and $\operatorname{gcd}(d, l)=1$ can be obtained from a $\Gamma_{0}(l)$ matrix, after one cancels off common factors of $c$ and $d$. 
Remark 3.12. Corollary 3.11 shows that each Fourier coefficient of $E_{k, i ; h}$ gives a function on the Jacobian of $X_{0}(l)$. However, these functions are clearly not holomorphic in general, else they would have to be constant.

Remark 3.13. An easy application of the Riemann-Roch theorem shows that the dimension of the space of $h$-twisted modular forms of weight $k \geq 2$ coincides with that of the space of usual modular forms of weight $k$. This holds for either $\Gamma_{0}(l)$ or $\Gamma_{1}(l)$, as well as for any nebentypus.

\section{Petersson inner Product For twisted MOdUlar Forms AND TWISTED EISENSTEIN SERIES}

In this section we define the Petersson inner product on the space of $h$-twisted modular forms of given weight $k$ and investigate its basic properties.

Definition 4.1. A $\Gamma_{1}(l)$-modular $h$-twisted form is called a cusp form, if in the second condition of Definition 3.1 the function

$$
f\left(\frac{a \tau+b}{c \tau+d}\right)(c \tau+d)^{-k}
$$

has limit 0 as $\tau \rightarrow$ i $\infty$ for all $\left(\begin{array}{cc}a & b \\ c & d\end{array}\right)$.

Definition 4.2. Let $f$ and $g$ be two $h$-twisted modular forms for $\Gamma_{1}(l)$ of weight $k$. Assume that at least one of then is a cusp form. We define Petersson inner product $\langle f, g\rangle$ by

$$
\langle f, g\rangle=\int_{\Gamma_{1}(l) \backslash \mathcal{H}} f(\tau) \overline{g(\tau)} y^{k-2} d x d y
$$

where $\tau=x+\mathrm{i} y$. The convergence is assured by the cusp condition.

Remark 4.3. We have to check that the above definition is independent of the choice of the fundamental domain. If $\tau=\frac{a \tau_{1}+b}{c \tau_{1}+d}$, then

$$
\begin{gathered}
2 \mathrm{i} y=\frac{a \tau_{1}+b}{c \tau_{1}+d}-\frac{a \bar{\tau}_{1}+b}{c \bar{\tau}_{1}+d}=\frac{\left(a \tau_{1}+b\right)\left(c \bar{\tau}_{1}+d\right)-\left(c \tau_{1}+d\right)\left(a \bar{\tau}_{1}+b\right)}{\left(c \tau_{1}+d\right)\left(c \bar{\tau}_{1}+d\right)}, \\
\frac{\tau_{1}-\bar{\tau}_{1}}{\left(c \tau_{1}+d\right)\left(c \bar{\tau}_{1}+d\right)}=\frac{2 \mathrm{i} y_{1}}{\left(c \tau_{1}+d\right)\left(c \bar{\tau}_{1}+d\right)}
\end{gathered}
$$

and

$$
d x d y=\frac{1}{\left(c \tau_{1}+d\right)^{2}\left(c \bar{\tau}_{1}+d\right)^{2}} d x_{1} d y_{1}
$$


SO

$$
\begin{aligned}
& f(\tau) \overline{g(\tau)} y^{k-2} d x d y=f\left(\frac{a \tau_{1}+b}{c \tau_{1}+d} \overline{)} \overline{\left(\frac{a \tau_{1}+b}{c \tau_{1}+d}\right)} \frac{y_{1}^{k-2}}{\left(c \tau_{1}+d\right)^{k}\left(c \bar{\tau}_{1}+d\right)^{k}} d x_{1} d y_{1}\right. \\
& =f\left(\tau_{1}\right) \mathrm{e}^{-2 \pi \mathrm{i} \Re H\left(-\frac{d}{c}\right)} \overline{g\left(\tau_{1}\right)} \mathrm{e}^{2 \pi \mathrm{i} \Re H\left(-\frac{d}{c}\right)} y_{1}^{k-2} d x_{1} d y_{1}=f\left(\tau_{1}\right) \overline{g\left(\tau_{1}\right)} y_{1}^{k-2} d x_{1} d y_{1} .
\end{aligned}
$$

Remark 4.4. It is clear that the above defined Petersson pairing restricts to a nondegenerate Hermitean form on the space of $h$-twisted cusp forms.

The following proposition extends the orthogonality of Eisenstein series and cusp forms (see $[\mathrm{L}$ ) to the $h$-twisted case, for the particular class of Eisenstein series considered in this paper.

Proposition 4.5. For $k \geq 3$ we have

$$
\left\langle E_{k, i ; h}, g\right\rangle=0
$$

for any weight $k$ h-twisted cusp form $g$ for $\Gamma_{1}(l)$.

Proof. It is enough to check that $\left\langle\hat{E}_{k, \chi ; h}, g\right\rangle=0$ for all characters $\chi$ : $(\mathbb{Z} / l \mathbb{Z})^{*} \rightarrow \mathbb{C}^{*}$. We can also assume that $g$ is an eigenform for $\Gamma_{0}$-action, as in Remark 3.4. Denote the character of $g$ by $\psi$. We have

$$
\left\langle\hat{E}_{k, \chi ; h}, g\right\rangle=\int_{\Gamma_{1}(l) \backslash \mathcal{H}} \sum_{\Gamma_{\infty} \backslash \Gamma_{0}(l)}(c \tau+d)^{-k} \chi(d) \mathrm{e}^{2 \pi \mathrm{i} \Re H\left(-\frac{d}{c}\right)} \overline{g(\tau)} y^{k-2} d x d y .
$$

We switch the order of integration and summation and then switch to a different domain for each term. Namely, we make a substitution $\tau=\frac{d \tau_{1}-b}{-c \tau_{1}+a}$ for each term of the $\hat{E}$-series. This gives

$$
\begin{gathered}
\left\langle\hat{E}_{k, \chi ; h}, g\right\rangle=\sum_{\Gamma_{\infty} \backslash \Gamma_{0}(l)} \int_{\Gamma_{1}(l) \backslash \mathcal{H}}\left(-c \tau_{1}+a\right)^{k} \chi(d) \mathrm{e}^{2 \pi \mathrm{i} \Re H\left(-\frac{d}{c}\right)} . \\
\cdot\left(-c \bar{\tau}_{1}+a\right)^{k} \mathrm{e}^{-2 \pi \mathrm{i} \Re H\left(-\frac{d}{c}\right)} \overline{\psi(d)} \overline{g\left(\tau_{1}\right)} \frac{y_{1}^{k-2} d x_{1} d y_{1}}{\left(-c \tau_{1}+a\right)^{k}\left(-c \bar{\tau}_{1}+a\right)^{k}} \\
=\sum_{\Gamma_{\infty} \backslash \Gamma_{0}(l)} \int_{\Gamma_{1}(l) \backslash \mathcal{H}} \chi(d) \overline{\psi(d)} \overline{g\left(\tau_{1}\right)} y_{1}^{k-2} d x_{1} d y_{1} .
\end{gathered}
$$

This allows us to rewrite the pairing as

$$
\sum_{\Gamma_{1}(l) \backslash \Gamma_{0}(l)} \chi(d) \overline{\psi(d)} \int_{\Gamma_{\infty} \backslash \mathcal{H}} \overline{g\left(\tau_{1}\right)} y_{1}^{k-2} d x_{1} d y_{1} .
$$


We then observe that $\Gamma_{\infty} \backslash \mathcal{H}$ can be thought of as $0 \leq x \leq 1, y \geq 0$ and for a fixed $y$

$$
\int_{\mathrm{i} y}^{1+\mathrm{i} y} \overline{g(\tau)} d x=\sum_{m>0} \bar{r}_{m} \mathrm{e}^{-2 \pi \mathrm{i} m y} \int_{x=0}^{x=1} \mathrm{e}^{-2 \pi \mathrm{i} m x} d x=0 .
$$

At the last step we used that $g$ has a Fourier expansion with a zero leading term. Indeed, we have $H(\mathrm{i} \infty)=0$, which implies that $g(\tau+$ $1)=g(\tau)$, and the $g(\mathrm{i} \infty)=0$ follows from $g$ being a cusp form.

\section{Rationality CONJECTURE}

The motivation behind our investigation of twisted Eisenstein series is that they might provide a tool for studying the Jacobian of the modular curve $X_{0}(l)$. We now recall that $X_{0}(l)$ comes equipped with the natural $\mathbb{Q}$-structure, which then gives a $\mathbb{Q}$-structure on its Jacobian. The following conjecture relates the rationality of the coefficients of twisted Eisensten series and the rationality of the point on the Jacobian.

Conjecture 5.1. The sheaf $\mathcal{F}_{0}(h)$ on $X_{0}(l)$ gives a rational point on the Jacobian if and only if for each $k$ there exists a nonzero formal power series $R_{k}(q)$ such that all coefficients of the $q$-series $E_{k, i ; h} R_{k}(q)$ are rational for all $i$. Equivalently, the ratios of $E_{k, i ; h}$ and $E_{k, j ; h}$ have rational Fourier coefficients for all $k, i$ and $j$, provided $E_{k, j ; h} \not \equiv 0$.

Remark 5.2. A stronger version of Conjecture 5.1 would require $R_{k}=$ $R^{k}$ for some series $R=R(q)$.

We devote the remainder of the section to the evidence in favor of Conjecture 5.1. Since the evidence is mostly circumstantial anyway, we will be somewhat sketchy and leave the details to the reader.

First of all, the if part of the conjecture holds up to a finite index subgroup as long as $E_{k, i ; h}$ do not have common zeroes for some $k>0$. Indeed, the existence of $R_{k}$ implies that the module over the ring of $\Gamma_{1}(l)$ modular forms which is generated by $E_{k, i ; h}$ is defined over $\mathbb{Q}$. By the usual correspondence between graded modules and sheaves we see that the invertible sheaf $\mathcal{F}(h) \otimes \mathcal{L}^{\otimes k}$ is defined over $\mathbb{Q}$. Since $\mathcal{L}$ is defined over $\mathbb{Q}$, we see that the invertible sheaf $\mathcal{F}(h)$ on $X_{1}(l)$ is defined over $\mathbb{Q}$. Denote $\pi: X_{1}(l) \rightarrow X_{0}(l)$ and recall that $\mathcal{F}(h)=\pi^{*} \mathcal{F}_{0}(h)$. Then

$$
\mathcal{F}_{0}^{\phi(l) / 2} \cong\left(\Lambda^{\phi(l) / 2} \pi_{*} \mathcal{F}(h)\right) \otimes\left(\Lambda^{\phi(l) / 2} \pi_{*} \pi^{*} \mathcal{O}\right)^{-1}
$$

is defined over $\mathbb{Q}$. 
For the only if part of the conjecture, observe that it holds for $h=0$, in view of Remark 2.10. In general, for every rational point on the Jacobian of $X_{1}(l)$ that corresponds to a sheaf $\mathcal{F}=\pi^{*} \mathcal{F}_{0}$, the space of global sections $H^{0}\left(\mathcal{F} \otimes L^{\otimes k}\right)$ has a basis that is defined over $\mathbb{Q}$. The essence of the Conjecture 5.1 is that the $h$-twisted Eisenstein series $E_{k, i ; h}$ are rational linear combinations of the basis elements. Since $q^{0}$ coefficients and the $\Gamma_{0}(l) / \Gamma_{1}(l)$ transformation properties of $E_{k, i ; h}$ are independent of the twisting, if the linear span of $E_{k, i ; h}$ is defined over rationals, then so are the individual elements, at least in the case of prime $l$. Indeed, one can show that in this case the matrix of $q^{0}$ coefficients of $\Gamma_{0}(l) / \Gamma_{1}(l)$-translates of $E_{k, i}$ is nondegenerate and rational. Consequently, if $\operatorname{Span}\left(E_{k, i ; h}\right)$ has a rational basis, $E_{k, i ; h}$ have rational coefficients in it.

Unfortunately, it is not at all obvious that $\operatorname{Span}\left(E_{k, i ; h}\right)$ is defined over $\mathbb{Q}$. The space of all $h$-twisted forms of weight $k$ splits up as a direct sum of the space of cusp forms and its orthogonal complement under the Petersson pairing. One can hope that this splitting respects the rational structures. In the case of prime $l$, the series $E_{k, i ; h}(\tau)$ roughly account for half the dimension of this orthogonal complement. However, if one could define $E_{k, i, h}(\tau)$ for weight $k=1$, then one would expect that all weight one $h$-twisted modular forms lie in $\operatorname{Span}\left(E_{k, i ; h}\right)$, by an application of Riemann-Roch formula.

On the plus side, it is quite easy to give a sufficient condition that assures that the Fourier coefficients of $\frac{1}{(2 \pi \mathrm{i})^{k}} E_{k, i ; h}$ are real. Recall that an $\mathbb{R}$-structure on the modular curve is given by the involution that sends $h(\tau)$ to $\overline{h(-\bar{\tau})}$. Let us calculate $\overline{E_{k, i ; h}(-\bar{\tau})}$.

Proposition 5.3. Let $g=\overline{h(-\tau)}$. For $k \geq 3$ one has

$$
\overline{E_{k, i ; h}(-\bar{\tau})}=(-1)^{k} E_{k, i ; g}(\tau)
$$

Proof. and let $G$ be the antiderivative $\int_{\mathrm{i} \infty}^{\tau} g(s) d s$ of $g$. Observe that $\overline{H(-\bar{\tau})}=-G(\tau)$. We have

$$
\begin{aligned}
& \overline{E_{k, i ; h}(-\bar{\tau})}=\sum_{c \in l \mathbb{Z}} \sum_{d \in \mathbb{Z}, \operatorname{gcd}(l, d)=1}(-c \tau+d)^{-k} \mathrm{e}^{2 \pi \mathrm{i} \frac{d i}{l}} \mathrm{e}^{-2 \pi \mathrm{i} \Re H\left(-\frac{d}{c}\right)} \\
& =\sum_{c \in l \mathbb{Z}} \sum_{d \in \mathbb{Z}, \operatorname{gcd}(l, d)=1}(-c \tau+d)^{-k} \mathrm{e}^{2 \pi \mathrm{i} \frac{d i}{l}} \mathrm{e}^{2 \pi \mathrm{i} \Re G\left(\frac{d}{c}\right)}=E_{k,-i ; g}(\tau)
\end{aligned}
$$

where we have switched from $c$ to $-c$ in the summation. It remains to observe $E_{k,-i ; g}=(-1)^{k} E_{k, i ; g}$, in view of the change from $(c, d)$ to $(-c,-d)$ in the summation. 
Corollary 5.4. If all Fourier coefficients of $h(\tau)$ are real, then all Fourier coefficients of $\frac{1}{(2 \pi \mathrm{i})^{k}} E_{k, i ; h}(\tau)$ are real.

Proof. Notice that If $F(\tau)=\sum_{m} r_{m} q^{m}$ then $\overline{F(-\bar{\tau})}=\sum_{m} \overline{r_{m}} q^{m}$. Then use the above proposition with $h=g$.

\section{Open PRoblems}

There are numerous open problems, whose solution would greatly enhance the understanding of the $h$-twisted modular forms. We list several of them.

- Can one efficiently calculate Fourier coefficients of an $h$-twisted Eisenstein series? The problem is that the definition only provides a relatively slowly convergent series. Lack of explicit coefficients makes it impossible to do any kind of computer experiments to uncover relations among the series or to test Conjecture 5.1

- Can one define $h$-twisted Eisenstein series for weights $k=1$ or 2? What will happen to their modularity properties? The similar question in the untwisted case is answered by looking at the Fourier expansions, but this approach seems to fail in general.

- In the untwisted case, these Eisenstein series can be constructed from logarithmic derivatives of the theta function, see [BG1]. Is there a twisted analog of the theta function, with the same properties? In a related comment, the notion of $h$-twisting can be extended to the Jacobi forms.

- Do any twisted forms have nice infinite product expansions?

- What is the meaning of Hecke operators in this setting? It is easy to see that the usual definition of the Hecke operators does not produce operators on the spaces of $h$-twisted forms. Also, if $h$ is a Hecke eigenform, does this lead to any properties of $h$ twisted forms? A related question is what should be the analog of Dirichlet series and Euler expansions in the $h$-twisted case.

- What is the meaning of the Fricke involution in this setting?

- What would be an analog of Rankin-Selberg method for the Petersson inner product of a usual cusp form and product of two twisted Eisenstein series of opposite twists? The argument of Proposition 4.5 shows that the unfolding trick generally works, but the lack of Euler expansions is a major hindrance.

- Is it possible to see what the twisted Eisenstein series are in the case of the divisors coming from the Heegner points? 
- What is the relation between the Eisenstein series of opposite twists?

- In the untwisted case, there are quadratic relations on the Eisenstein series that mimic the relations on modular symbols. Is there any analog of such relations in the twisted case? For instance, dimension counts show that there are a lot of linear relations between products of $h$-twisted and untwisted Eisenstein series. Can one write at least some of them explicitly? Together with the rationality conjecture, this might provide an approach to the Birch-Swinnerton-Dyer conjecture, see [T], although at this point in time this is, at best, a very long shot.

\section{REFERENCES}

[BG1] L. Borisov, P. Gunnells, Toric varieties and modular forms. Invent. Math. 144 (2001), no. 2, 297-325.

[BG2] L. Borisov, P. Gunnells, Toric modular forms of higher weight. J. Reine Angew. Math. 560 (2003), 43-64.

[Go] D. Goldfeld, Zeta functions formed with modular symbols. Automorphic forms, automorphic representations, and arithmetic (Fort Worth, TX, 1996), 111-121, Proc. Sympos. Pure Math., 66, Part 1, Amer. Math. Soc., Providence, RI, 1999.

[GG] D. Goldfeld, P. Gunnells, Eisenstein series twisted by modular symbols for the group $\mathrm{SL}_{n}$. Math. Res. Lett. 7 (2000), no. 5-6, 747-756.

[GH] P. Griffiths, J. Harris, Principles of algebraic geometry. Reprint of the 1978 original. Wiley Classics Library. John Wiley \& Sons, Inc., New York, 1994.

[L] S. Lang, Introduction to modular forms. Grundlehren der mathematischen Wissenschaften, No. 222. Springer-Verlag, Berlin-New York, 1976.

[P] Y. Petridis, Spectral deformations and Eisenstein series associated with modular symbols. Int. Math. Res. Not. 2002, no. 19, 991-1006.

[T] J. Tate, On the conjectures of Birch and Swinnerton-Dyer and a geometric analog. Séminaire Bourbaki, Vol. 9, Exp. No. 306, 415-440, Soc. Math. France, Paris, 1995. 11G40 (14G10)

Department of Mathematics, University of Wisconsin, Madison, Wi, 53706, USA, borisov@math.wisc.edu 